\title{
Continuous Collision Detection for Two Moving Elliptic Disks
}

\author{
Yi-King Choi, Wenping Wang, Yang Liu, and Myung-Soo Kim
}

\begin{abstract}
Collision detection and avoidance are important in robotics. Compared with commonly used circular disks, elliptic disks provide a more compact shape representation for robots or other vehicles confined to move in the plane. Furthermore, elliptic disks allow a simpler analytic representation than rectangular boxes, which makes it easier to perform continuous collision detection (CCD). We shall present a fast and accurate method for CCD between two moving elliptic disks, which avoids any need to sample the time domain of the motion, thus avoiding the possibility of missing collisions between time samples. Based on some new algebraic conditions on the separation of two ellipses, we reduce collision detection for two moving ellipses to the problem of detecting real roots of a univariate equation, which is the discriminant of the characteristic polynomial of the two ellipses. Several techniques are investigated for robust and accurate processing of this univariate equation for two classes of commonly used motions: planar cycloidal motions and planar rational motions. Experimental results demonstrate the efficiency, accuracy, and robustness of our method.
\end{abstract}

Index Terms-Collision detection, ellipses, elliptic disks, interference analysis, rational motion.

\section{INTRODUCTION}

C OLLISION detection is important in robotics for path planning and simulation. Accurate collision-detection algorithms can greatly facilitate the avoidance of collisions between moving objects. In applications where real-time response is mandatory, efficient collision-detection algorithms are also essential. Although many collision-detection algorithms cater to 3-D applications [1], [2], there are numerous other applications in which objects only move in the plane. Examples are robot or vehicle path planning, where the robots or vehicles are represented by 2-D figures and move in the 2-D plane, but the robots may follow any path and make arbitrary motions.

Even in a 2-D setting, the outline of an object can be quite complicated, and a two-phase approach to collision detection is widely adopted in practice. Objects are enclosed by simple geometric entities, called bounding objects, to which simpler collision detection is first applied; more complicated collision-detection computation on the detailed objects will only be carried out if their bounding objects are found to be overlapping.

Manuscript received December 11, 2004; revised June 11, 2005. This paper was recommended for publication by Associate Editor P. Rocco and Editor S. Hutchinson upon evaluation of the reviewers' comments. This work was supported in part by the Research Grant Council of Hong Kong SAR under Grant HKU7153/03E, in part by the National Key Basic Research Project of China under 2004CB318000, and in part by the Korean Ministry of Information and Communication under the Program of IT Research Center on CGVR.

Y.-K. Choi, W. Wang, and Y. Liu are with the Department of Computer Science, University of Hong Kong, Hong Kong (e-mail: ykchoi@cs.hku.hk).

M.-S. Kim is with the School of Computer Science and Engineering, Seoul National University, Seoul 151-742, Korea.

Digital Object Identifier 10.1109/TRO.2005.862479
Commonly used bounding objects include circular disks and rectangles. There are, in general, two criteria in choosing the type of bounding objects to be used in a particular application. The first is bounding tightness: bounding objects should be as tight as possible so that when two enclosed objects are separate, their bounding objects should also be separate for most of the time. This criterion saves time by ensuring that many noncolliding pairs are not subject to further processing once their bounding objects are found to be separate. The second criterion is that collision detection for a pair of bounding objects should be simple and very fast, since this operation usually needs to be done many times, i.e., for every pair of objects present in an environment. From these considerations, it is not hard to understand why circular discs are commonly used as bounding objects for robots in the plane (e.g., [3]); collision detection between a pair of circles is almost trivial, and can therefore be performed very efficiently. Interference testing of multiple circular discs has also been studied intensively in computational geometry [4]-[6]. (Spheres are popular bounding objects in the 3-D case, for similar reasons.)

Ellipses provide much tighter bounding than circles. When ellipses or circles are used as bounding objects, far fewer ellipses than circles are normally needed to enclose a given object with the same degree of tightness. Therefore, the use of ellipses as bounding objects can potentially lead to significant improvement in the accuracy and efficiency of collision detection. However, relatively little work can be found in the literature on the use of ellipses as bounding objects, largely because of the lack of effective means of collision detection for ellipses. There are several major issues in using ellipses as bounding objects, which include computing the smallest bounding ellipse for a given object, detecting the collision of two moving ellipses, and computing the penetration distance of two overlapping ellipses. In this paper, we shall focus on collision detection between two elliptic disks with prespecified continuous motions.

Thorough analysis and classification of intersection of general conics can be found in classical algebraic geometry, e.g., [7]-[9]. These results, however, consider conics in the complex (projective, affine, or Euclidean) plane, and are not applicable to the 2-D collision-detection problem, for which the analysis must be done in the real plane. There is, nevertheless, an obvious way to detect intersection between stationary ellipses, which is to compute their real intersection points. But this brute-force approach entails the numerical solution of a quartic equation, and is, therefore, difficult to extend to deal with moving ellipses. In this paper, we establish a simple condition for checking the separation of two ellipses in the real plane based on the number of the real roots of their characteristic equation, and apply it to collision detection of two moving ellipses. To the best of our knowledge, this is a new result that is unknown in classical geometry. 
A typical framework for collision detection between moving objects is to sample the time interval of the motion at discrete time instants and test whether the objects intersect at each sampled instant. This temporal sampling approach is prone to error, since it may miss collisions that occur between sample instants. A recent work by Schwarzer et al. [10] uses adaptive sampling, which guarantees that all configurations along a straight-line segment connecting two configurations in the $\mathrm{C}$-space are collision-free, by considering also the distance information in the workspace. Based on this technique, Ferré and Laumond [11] further developed a collision-free planar path through an iterative process. There have also been attempts to use the speed of moving objects as a bound to determine the safe time-sampling resolution [12]. In recent work [13]-[15], Redon and his colleagues address the important issue of continuous collision detection (CCD) in various computing environments, which include hundreds of thousands of polygons as obstacles and complex moving objects, such as those composed of articulated links. In particular, Redon et al. [14], [15] apply a graphics processing unit (GPU)-based collision detection to the swept volumes of line swept sphere (LSS) primitives against the environment. On the other hand, our current work takes a different approach and emphasizes the use of algebraic conditions.

We shall present a fast and accurate algorithm for CCD between two moving ellipses in the plane. We introduce new conditions on the separation of two ellipses to reduce the collision-detection problem to the problem of detecting a real zero of a univariate function which is the discriminant of the characteristic polynomial of the two ellipses. If the ellipses serve as bounding objects, the colliding time intervals computed by our method can then be used as a refined time span to which other algorithms for collision detection on the exact objects may be applied.

Our method is based on theoretical results similar to those of Wang et al. in [16], concerning the separation of two stationary ellipsoids in 3-D space, but there are important differences between these results for ellipses and ellipsoids. First, the separation condition for two stationary ellipses cannot be derived as a special case of the result [16] for two stationary ellipsoids, although the former is a low-dimensional counterpart of the latter. Therefore, in this paper, we shall prove, for the first time, an algebraic condition on the separation of two stationary ellipses. Second, compared with ellipsoids, the characteristic polynomial of two ellipses has relatively simple properties, and this simplicity allows us to reduce collision detection in the moving case to a problem of detecting the zero of a univariate function. In general, such a treatment is not possible for two moving ellipsoids, at least not in the same straightforward manner, as will be discussed in detail later. Our approach to collision detection between moving ellipsoids [17] addresses this issue, and is based on the zero-set analysis of a bivariate function.

The contributions of this paper can be summarized as follows.

1) A simple algebraic condition is established for the separation of two stationary ellipses.

2) An algebraic condition is established for detecting collisions between two moving ellipses.

3) An algorithmic framework is presented for fast and accurate collision detection between two moving ellipses. We discuss in detail two classes of commonly used motions: cycloidal motions and rational motions. We also present robust methods for processing high-degree polynomials arising from the use of rational motions, which contribute to reliable collision detection.

The remainder of this paper is organized as follows. In Section II, we shall establish the algebraic condition for the separation of two stationary ellipses, and other algebraic properties of the configuration formed by a pair of ellipses. These results are then used in Section III to prove a separation condition for two moving ellipses. The framework for our collision-detection algorithm is presented in Section IV. In Section V, we discuss details of the formulation and processing of the cycloidal motion, which is a simple but commonly used nonrational motion. In Section VI, we present algorithms for ellipses making rational motions, concentrating on devising numerically stable algorithms for processing the high-degree polynomials that arise from rational motions. We conclude the paper and discuss further research problems in Section VII.

\section{Condition on Separation of Two Ellipses}

In this section, we will prove the separation condition for two stationary ellipses. An ellipse is a conic section curve, and can be represented in the Euclidean plane $\mathbb{E}^{2}$ by $X^{T} A X=0$, where $A=\left[a_{i, j}\right]$ is a $3 \times 3$ real symmetric matrix, and $X$ is a 3-D column vector containing the homogeneous coordinates of a point in $\mathbb{E}^{2}$. Let $A_{i, i}$ denote the leading submatrix of size $i \times i$ of $A, i=1,2,3$. For an ellipse $X^{T} A X=0$, we shall assume throughout that the matrix $A$ is normalized such that $\bar{X}^{T} A \bar{X}<0$ for any interior point $\bar{X}$ of the ellipse. Then, by elementary geometry, an ellipse $X^{T} A X=0$ is characterized by the conditions that $\operatorname{det}\left(A_{1,1}\right)=a_{1,1}>0, \operatorname{det}\left(A_{2,2}\right)>0$, and $\operatorname{det}\left(A_{3,3}\right)<0$. Thus, $A_{2,2}$ is positive definite.

An elliptic disk $\mathcal{A}$ is defined by $\mathcal{A} \equiv\left\{X \mid X^{T} A X \leq 0\right\} \subset$ $\mathbb{E}^{2}$. We use $\partial \mathcal{A}$ to denote the boundary curve of $\mathcal{A}$, i.e., the set of points satisfying $X^{T} A X=0$, and use $\operatorname{Int}(\mathcal{A})$ to denote the interior points of $\mathcal{A}$. Thus, $\mathcal{A}=\partial \mathcal{A} \cup \operatorname{Int}(\mathcal{A})$. For brevity, we will use the terms ellipse and elliptic disk interchangeably when there is no danger of confusion.

Two elliptic disks, $\mathcal{A}: X^{T} A X \leq 0$ and $\mathcal{B}: X^{T} B X \leq 0$, are said to be separate or disjoint if $\overline{\mathcal{A}} \cap \mathcal{B}=\emptyset$. The disks $\mathcal{A}$ and $\mathcal{B}$ are said to be overlapping if $\operatorname{Int}(\mathcal{A}) \bigcap \operatorname{Int}(\mathcal{B}) \neq \emptyset$; and they are said to be touching if $\mathcal{A} \cap \mathcal{B} \neq \emptyset$ and $\operatorname{Int}(\mathcal{A}) \bigcap \operatorname{Int}(\mathcal{B})=\emptyset$ (see Fig. 1).

Given two elliptic disks $\mathcal{A}: X^{T} A X \leq 0$ and $\mathcal{B}: X^{T} B X \leq$ 0 , the cubic polynomial $\mathrm{f}(\lambda)=\operatorname{det}(\lambda A-B)$ is called the characteristic polynomial, and $\mathrm{f}(\lambda)=0$ the characteristic equation of $\mathcal{A}$ and $\mathcal{B}$.

Lemma 1: For any two elliptic disks $\mathcal{A}: X^{T} A X \leq 0$ and $\mathcal{B}: X^{T} B X \leq 0$, the root pattern of $\mathrm{f}(\lambda)=0$ falls into one of the following three cases:

1) three positive roots;

2) one positive and two negative roots;

3) one positive and a pair of complex conjugate roots.

Proof: Suppose

$$
f(\lambda)=a_{3} \lambda^{3}+a_{2} \lambda^{2}+a_{1} \lambda+a_{0} .
$$

Then $a_{3}=\operatorname{det}(A)<0$ and $a_{0}=-\operatorname{det}(B)>0$. It follows that $\mathrm{f}(0)>0$ and $\mathrm{f}(+\infty)<0$. Hence, $\mathrm{f}(\lambda)=0$ has at least one positive root. Moreover, since $a_{3} \neq 0$ and $a_{0} \neq 0$, it is clear that 


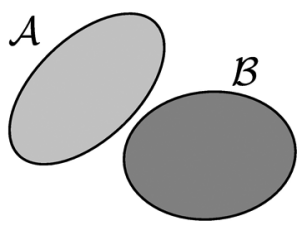

(a) $\mathcal{A} \cap \mathcal{B}=\emptyset$

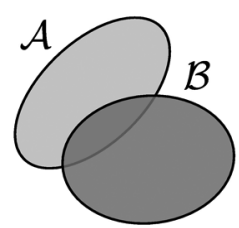

(b) $\operatorname{Int}(\mathcal{A}) \cap$ $\operatorname{Int}(\mathcal{B}) \neq$

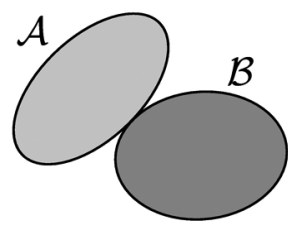

(c) $\mathcal{A} \cap \mathcal{B} \neq \emptyset$ and $\operatorname{Int}(\mathcal{A}) \cap \operatorname{Int}(\mathcal{B})=\emptyset$
Fig. 1. Two elliptic disks $\mathcal{A}$ and $\mathcal{B}$. (a) Separate. (b) Overlapping. (c) Touching.

0 or $\infty$ cannot be a root of $\mathrm{f}(\lambda)=0$. Let $\lambda_{0}>0, \lambda_{1}$, and $\lambda_{2}$ denote the three roots. Since $\lambda_{0} \lambda_{1} \lambda_{2}=-a_{0} / a_{3}>0$, we have $\lambda_{1} \lambda_{2}>0$. Hence, the other two roots $\lambda_{1}$ and $\lambda_{2}$ must both be positive, both negative, or a pair of complex conjugates.

Lemma 2: If $\operatorname{Int}(\mathcal{A}) \bigcap \operatorname{Int}(\mathcal{B})=\emptyset$, then $\mathrm{f}(\lambda)=0$ has a negative root.

Proof: Since $\operatorname{Int}(\mathcal{A}) \bigcap \operatorname{Int}(\mathcal{B})=\emptyset$, we may suppose that $\mathcal{A}$ and $\mathcal{B}$ are either separate or touching externally. We make the substitution $\lambda=(\mu-1) / \mu$, which maps $\mu \in[0,1]$ to $\lambda \in(-\infty, 0]$, and transforms the characteristic equation $\mathrm{f}(\lambda)=$ $\operatorname{det}(\lambda A-B)=0$ to $\mathrm{g}(\mu) \equiv \operatorname{det}((1-\mu) A+\mu B)=0$. We will denote $Q(\mu) \equiv(1-\mu) A+\mu B$, observing that $Q(0)=A$ and $Q(1)=B$. Clearly, $\mathrm{f}(\lambda)=0$ has a finite negative root if and only if (iff) $\mathrm{g}(\mu)=0$ has a real root in $(0,1)$. We shall now show by contradiction that $\mathrm{g}(\mu)=0$ has a real root in $(0,1)$.

Assume that $\mathrm{g}(\mu)=0$ has no real root in $(0,1)$. Since $\mathrm{g}(\mu) \equiv$ $\operatorname{det}((1-\mu) A+\mu B)$ is a continuous function of $\mu$, and $\mathrm{g}(0)=$ $\operatorname{det}(A)<0$, we have $\mathrm{g}(\mu)=\operatorname{det}((1-\mu) A+\mu B)<0$ for all $\mu \in[0,1]$. (Recall that $\mathrm{g}(1)=\operatorname{det}(B) \neq 0$.) Clearly, $\operatorname{det}\left(Q(\mu)_{1,1}\right)=(1-\mu) a_{1,1}+\mu b_{1,1}>0$ for all $\mu \in[0,1]$, since $A_{1,1}>0$ and $B_{1,1}>0$. Furthermore, $Q(\mu)_{2,2}=(1-$ н) $A_{2,2}+\mu B_{2,2}$ is positive definite for any $t \in[0,1]$, since $A_{2,2}$ and $B_{2,2}$ are positive definite. Thus, $\operatorname{det}\left(Q(\mu)_{2,2}\right)>0$ for all $\mu \in[0,1]$. Hence, $X^{T} Q(\mu) X=0$ is an ellipse for all $\mu \in[0,1]$, with its center at $R(\mu)=Q(\mu)^{-1}[0,0,1]^{T}$.

Let us denote $\mathrm{p}(\mu) \equiv R(\mu)^{T} A R(\mu)$. Then $\mathrm{p}(\mu)$ is a continuous function of $\mu$ in $[0,1]$. Clearly, $R(0) \in \operatorname{Int}(\mathcal{A})$, since $R(0)$ is the center of $\mathcal{A}$. We have $R(1) \notin \mathcal{A}$, since $R(1)$ is the center of $\mathcal{B}$ and $\operatorname{Int}(\mathcal{A}) \cap \operatorname{Int}(\mathcal{B})=\emptyset$, which is the hypothesis of the lemma. Hence, $\mathrm{p}(0)=R(0)^{T} A R(0)<0$ and $\mathrm{p}(1)=R(1)^{T} A R(1)>0$. By a continuity argument, it follows that $\mathrm{p}\left(\mu_{1}\right)=0$ for some $\mu_{1} \in[0,1]$, i.e., the center $R\left(\mu_{1}\right)$ of the ellipse $\mathcal{Q}\left(\mu_{1}\right)$ is on the boundary of the elliptic disk $\mathcal{A}$ (see Fig. 2). We then define a circular disk $\mathcal{D}$ centered at $R\left(\mu_{1}\right)$ which is contained in $\mathcal{Q}\left(\mu_{1}\right)$, i.e., $\mathcal{D} \subset \mathcal{Q}\left(\mu_{1}\right)$. Note that $R\left(\mu_{1}\right)$ may or may not be the tangent point of $\mathcal{A}$ and $\mathcal{B}$, since $\mathcal{A}$ and $\mathcal{B}$ are either separate or touching externally. If $R\left(\mu_{1}\right)$ is not the tangent point, $\mathcal{D}$ can be made sufficiently small such that $\mathcal{D} \cap \mathcal{B}=\emptyset$. In any case, the tangent of $\mathcal{A}$ at $R\left(\mu_{1}\right)$ (also the common tangent of $\mathcal{A}$ and $\mathcal{B}$ at $R\left(\mu_{1}\right)$, if $R\left(\mu_{1}\right)$ is the tangent point) gives a diameter of the disk $\mathcal{D}$. We may then choose a point $X_{1}$ on this diameter, except for $R\left(\mu_{1}\right)$, such that $X_{1}$ is interior to $\mathcal{Q}\left(\mu_{1}\right)$, but is exterior to both $\mathcal{A}$ and $\mathcal{B}$. Hence, we have $X_{1}^{T} Q\left(\mu_{1}\right) X_{1}<0, X_{1}^{T} A X_{1}>0$, and $X_{1}^{T} B X_{1}>0$. Since $\mu_{1} \in(0,1)$, it follows that

$$
X_{1}^{T} Q\left(\mu_{1}\right) X_{1}=\left(1-\mu_{1}\right) X_{1}^{T} A X_{1}+\mu_{1} X_{1}^{T} B X_{1}>0 .
$$

This is a contradiction. Hence, $\mathrm{g}(\mu)$ has a real zero in $(0,1)$.

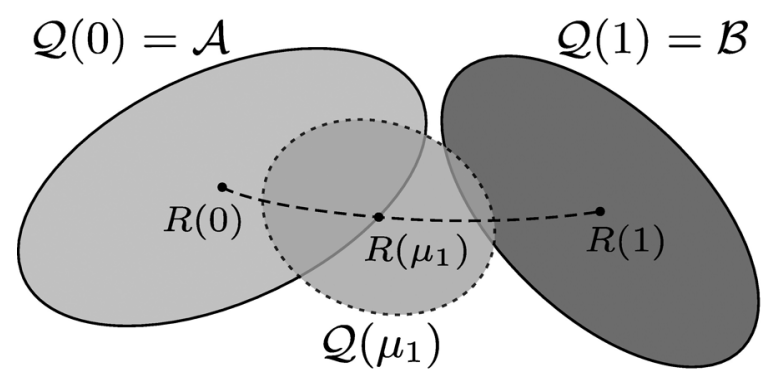

Fig. 2. Configuration for Lemma 2.

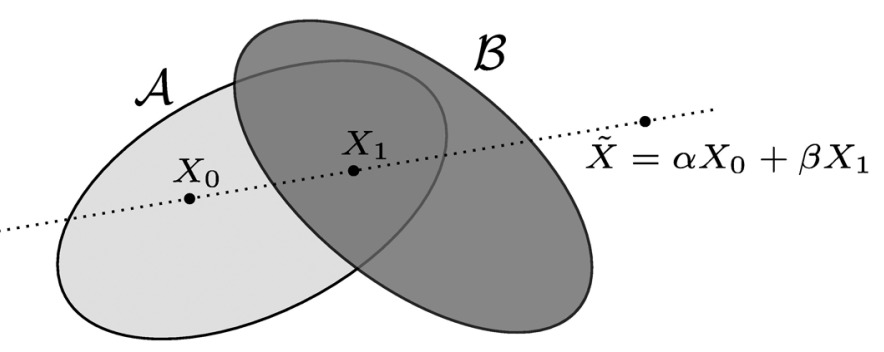

Fig. 3. Configuration for Lemma 3.

Lemma 3: If $\operatorname{Int}(\mathcal{A}) \bigcap \operatorname{Int}(\mathcal{B}) \neq \emptyset$, then any real root of $\mathrm{f}(\lambda)=0$ is positive.

Proof: The proof goes by contradiction. Let $\lambda_{0}$ be a real root of $\mathrm{f}(\lambda)=0$. Assume $\lambda_{0} \leq 0$ and denote $Q_{0}=\lambda_{0} A-B$. Then there exists a real point $X_{0}$ such that $Q_{0} X_{0}=0$, because $Q_{0}$ is singular. Since $\operatorname{Int}(\mathcal{A}) \cap \operatorname{Int}(\mathcal{B}) \neq \emptyset$, let $X_{1}\left(\neq X_{0}\right)$ denote a common interior point of $\mathcal{A}$ and $\mathcal{B}$, i.e., $X_{1}^{T} A X_{1}<0$ and $X_{1}^{T} B X_{1}<0$ (Fig. 3). Then

$$
X_{1}^{T} Q_{0} X_{1}=\lambda_{0} X_{1}^{T} A X_{1}-X_{1}^{T} B X_{1}>0 .
$$

Let $\mathcal{L}$ denote the line passing through $X_{0}$ and $X_{1}$. Then, since $\mathcal{A}$ and $\mathcal{B}$ are bounded, there exists on the line $\mathcal{L}$ a point $\tilde{X}$ far enough from $\mathcal{A}$ and $\mathcal{B}$ such that $\tilde{X}$ is exterior to both $\mathcal{A}$ and $\mathcal{B}$. Let us write $\tilde{X}=\alpha X_{0}+\beta X_{1}$, where $\alpha$ and $\beta$ are real constants that are not both zero. Then $\tilde{X}^{T} A \tilde{X}>0$ and $\tilde{X}^{T} B \tilde{X}>0$. It follows that

$$
\tilde{X}^{T} Q_{0} \tilde{X}=\lambda_{0} \tilde{X}^{T} A \tilde{X}-\tilde{X}^{T} B \tilde{X}<0 .
$$

On the other hand, since $Q_{0} X_{0}=0$ and $X_{1}^{T} Q_{0} X_{1}>0$, we have

$$
\begin{aligned}
\tilde{X}^{T} Q_{0} \tilde{X} & =\alpha^{2} X_{0}^{T} Q_{0} X_{0}+2 \alpha \beta X_{1}^{T} Q_{0} X_{0}+\beta^{2} X_{1}^{T} Q_{0} X_{1} \\
& =\beta^{2} X_{1}^{T} Q_{0} X_{1} \geq 0 .
\end{aligned}
$$

This is a contradiction. Hence, any real root $\lambda_{0}$ of $f(\lambda)=0$ is positive.

Lemma 4: If two elliptic disks $\mathcal{A}: X^{T} A X \leq 0$ and $\mathcal{B}$ : $X^{T} B X \leq 0$ touch externally, then $\mathrm{f}(\lambda)=0$ has a negative double root.

Proof: Suppose that $\mathcal{A}$ and $\mathcal{B}$ do touch externally. As a result, the two ellipses $X^{T} A X=0$ and $X^{T} B X=0$ will have a multiple intersection. Then $\mathrm{f}(\lambda)=0$ has a multiple root $\lambda_{0}$ $[18$, p. 256]. Since $\operatorname{Int}(\mathcal{A}) \cap \operatorname{Int}(\mathcal{B})=\emptyset$, by Lemma $2, \mathrm{f}(\lambda)=$ 0 has a negative root $\lambda_{1}$. Moreover, by Lemma $1, \mathrm{f}(\lambda)=0$ has a positive root $\lambda_{2}$. Thus, we have either $\lambda_{0}=\lambda_{1}<0$ or $\lambda_{0}=\lambda_{2}>0$. Again by Lemma 1 , only the first case is possible. Hence, $f(\lambda)=0$ has a negative double root. 
Lemma 5: If $\mathrm{f}(\lambda)=0$ has a negative double root, then the elliptic disks $\mathcal{A}: X^{T} A X \leq 0$ and $\mathcal{B}: X^{T} B X \leq 0$ touch each other externally. Moreover, the real touching point $X_{0}$ is the unique solution to $\left(\lambda_{0} A-B\right) X=0$, where $\lambda_{0}$ is the negative double root of $\mathrm{f}(\lambda)=0$.

Proof: Let $\lambda_{0}<0$ be a negative double root of $\mathrm{f}(\lambda)=0$. Clearly, $\lambda_{0}$ is not a zero of the first $2 \times 2 \operatorname{minors} \operatorname{det}\left(\lambda A_{2,2}-\right.$ $B_{2,2}$ ), because both $A_{2,2}$ and $B_{2,2}$ are positive definite, and thus the two zeros of $\operatorname{det}\left(\lambda A_{2,2}-B_{2,2}\right)$ are positive. It follows that rank $\left(\lambda_{0} A-B\right)=2$ and its null space, $\operatorname{Ker}\left[\lambda_{0} A-B\right]$, has dimension one.

Since $\operatorname{det}(\lambda A-B)=0$ has a double root $\lambda_{0}$, the pencil $X^{T}(\lambda A-B) X=0$ contains the singular conic $\lambda_{0} A-B$ with multiplicity 2 . In this case, the two ellipses $X^{T} A X=0$ and $X^{T} B X=0$ are tangential to each other at the singular point $X_{0}$ of the conic $X^{T}\left(\lambda_{0} A-B\right) X=0$, that is, $\left(\lambda_{0} A-B\right) X_{0}=0$ (see [7]).

We are now going to show that $X_{0}$ is a real tangent point of $\mathcal{A}$ and $\mathcal{B}$. Let us suppose that $X_{0}=U \pm i V \neq 0$, where $U$ and $V$ are real homogeneous vectors which are not both zero; without loss of generality, we suppose that $U \neq 0$. Then, from $\left(\lambda_{0} A-B\right)(U \pm i V)=0$, it follows that $\left(\lambda_{0} A-B\right) U=0$ and $\left(\lambda_{0} A-B\right) V=0$. This means that $U$ and $V$ are both real solutions of $\left(\lambda_{0} A-B\right) X=0$. Hence, $U$ and $V$ are linearly dependent, or $V=\alpha U$ for some constant $\alpha$, since $\operatorname{Ker}\left[\lambda_{0} A-B\right]$ has dimension one. It follows that $X_{0}=(1+i \alpha) U$ is a real point, since $U$ stands for a real point and the multiplicative constant $(1+i \alpha)$ can be ignored in a homogeneous representation. Hence, the elliptic disks $\mathcal{A}$ and $\mathcal{B}$ touch each other externally at the real point $X_{0}$.

Lemma 5 also suggests a convenient means to compute the contact point of two externally touching ellipses, which will be used to find the first contact point of two moving ellipses in subsequent sections.

The following theorem gives a condition on the separation of two elliptic disks, which is the main result of this section. Fig. 4 illustrates the relationship between two ellipses and the root pattern of their characteristic polynomial.

Theorem 6: Given two ellipses $\mathcal{A}: X^{T} A X=0$ and $\mathcal{B}$ : $X^{T} B X=0$ :

1) $\mathcal{A}$ and $\mathcal{B}$ touch externally iff $\mathrm{f}(\lambda)=0$ has a negative double root;

2) $\mathcal{A}$ and $\mathcal{B}$ are separate iff $\mathrm{f}(\lambda)=0$ has two distinct negative roots.

Proof: Part 1) follows from Lemmas 4 and 5. For part 2), the sufficiency follows from Lemmas 3 and 4, and the necessity follows from Lemmas 2 and 5.

Remark: The application of the above conditions to detecting the overlapping of two stationary ellipses is rather straightforward. A description of the resulting algorithm will be discussed in Section IV as the first step of our complete algorithm for moving ellipses. Note that a quick and exact test for overlap between two stationary ellipses should be of interest in its own right in some applications.

The next corollary, following from Theorem 6 2) and Lemma 1 , is a key property that enables us to detect collisions between
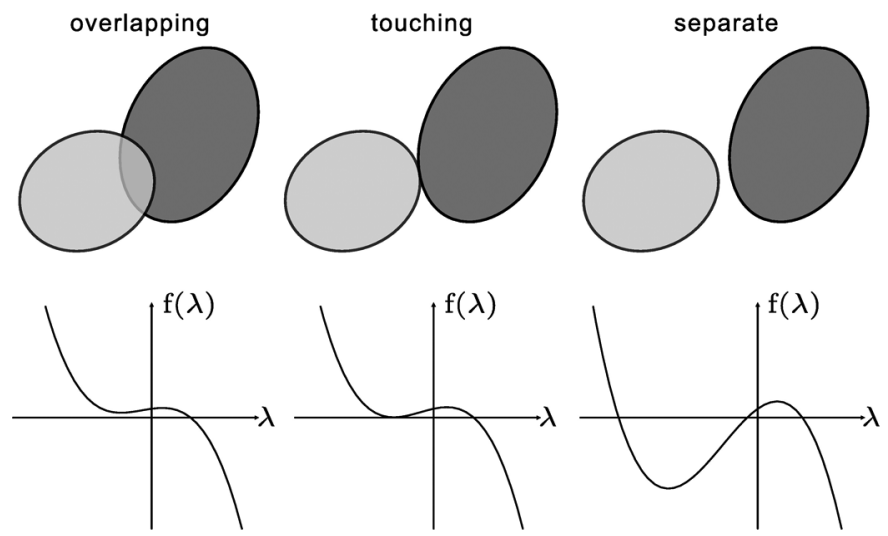

Fig. 4. Two elliptic disks and their characteristic polynomial $f(\lambda)$. Left: Overlapping iff $\mathrm{f}(\lambda)=0$ has no negative root. Middle: Touching externally iff $\mathrm{f}(\lambda)=0$ has a double negative root. Right: Separate iff $\mathrm{f}(\lambda)=0$ has two distinct negative roots.

two moving elliptic disks $\mathcal{A}(t)$ and $\mathcal{B}(t)$ by the occurrence of a double root of $\mathrm{f}(\lambda ; t)=0$, as will be seen in the next section.

Corollary 7: Suppose that two elliptic disks $\mathcal{A}: X^{T} A X \leq$ 0 and $\mathcal{B}: X^{T} B X \leq 0$ are separate. Then $\mathrm{f}(\lambda)=0$ does not have any multiple roots.

\section{Separation Condition For Two MOVING ELLIPTIC DISKS}

In this section, we are going to establish a condition for detecting a collision between two moving elliptic disks. Consider two elliptic disks $\mathcal{A}(t): X^{T} A(t) X \leq 0$ and $\mathcal{B}(t): X^{T} B(t) X \leq 0$ making continuous motions $\bar{M}_{A}(t)$ and $M_{B}(t), t \in[0,1]$ respectively. The disks $\mathcal{A}(t)$ and $\mathcal{B}(t)$ are said to be collision-free if $\mathcal{A}(t)$ and $\mathcal{B}(t)$ are separate for all $t \in[0,1]$. Otherwise, $\mathcal{A}(t)$ and $\mathcal{B}(t)$ collide, i.e., $\mathcal{A}(t)$ and $\mathcal{B}(t)$ are either touching or overlapping for some $t \in[0,1]$.

The characteristic polynomial of $\mathcal{A}(t)$ and $\mathcal{B}(t), t \in[0,1]$ is

$$
\mathrm{f}(\lambda ; t)=\operatorname{det}(\lambda A(t)-B(t))
$$

and we can write

$$
\mathrm{f}(\lambda ; t)=\mathrm{g}_{3}(t) \lambda^{3}+\mathrm{g}_{2}(t) \lambda^{2}+\mathrm{g}_{1}(t) \lambda+\mathrm{g}_{0}(t) .
$$

The discriminant of $\mathrm{f}(\lambda ; t)$ with respect to $\lambda$, as a function of $t$, is

$$
\Delta(t)=18 \mathrm{~g}_{3} \mathrm{~g}_{2} \mathrm{~g}_{1} \mathrm{~g}_{0}-4 \mathrm{~g}_{2}^{3} \mathrm{~g}_{0}+\mathrm{g}_{2}^{2} \mathrm{~g}_{1}^{2}-4 \mathrm{~g}_{3} \mathrm{~g}_{1}^{3}-27 \mathrm{~g}_{3}^{2} \mathrm{~g}_{0}^{2}
$$

(see [19]). By definition, $\mathrm{f}(\lambda ; t)=0$ has a multiple root in $\lambda$ for some $t$ iff $\Delta(t)=0$. Furthermore, it can be shown that $\mathrm{f}(\lambda ; t)=$ 0 has three simple real roots if $\Delta(t)>0$, and $\mathrm{f}(\lambda ; t)=0$ has two complex conjugate roots and a real root if $\Delta(t)<0$.

The next theorem states the condition that two moving elliptic disks are collision-free.

Theorem 8: Let $\mathcal{A}(t)$ and $\mathcal{B}(t), t \in[0,1]$ be two moving elliptic disks in $\mathbb{E}^{2}$. Let $\mathrm{f}(\lambda ; t)$ be their characteristic polynomial. Let $\Delta(t)$ denote the discriminant of $\mathrm{f}(\lambda ; t)$ with respect to $\lambda$. Suppose that $\mathcal{A}(0)$ and $\mathcal{B}(0)$ are separate. Then $\mathcal{A}(t)$ and $\mathcal{B}(t)$ are collision-free iff $\Delta(t)$ has no real zero in $[0,1]$.

Proof: First, we prove necessity. Suppose that $\mathcal{A}(t)$ and $\mathcal{B}(t)$ are collision-free. Then by Corollary $7, \mathrm{f}(\lambda ; t)=0$ does 
not have a multiple root in $\lambda$ for any $t \in[0,1]$. Therefore, $\Delta(t)$ does not have any real zero in $[0,1]$.

To prove sufficiency, suppose that $\Delta(t)=0$ has no real root in $[0,1]$. Now assume that $\mathcal{A}(t)$ and $\mathcal{B}(t)$ collide. Then $\mathcal{A}\left(t_{0}\right)$ and $\mathcal{B}\left(t_{0}\right)$ are overlapping or touching for some $t_{0} \in[0,1]$. Since $\mathcal{A}(0)$ and $\mathcal{B}(0)$ are separate, by a continuity argument, there exists an instant $t_{1} \in\left(0, t_{0}\right] \subset[0,1]$ at which $\mathcal{A}\left(t_{1}\right)$ and $\mathcal{B}\left(t_{1}\right)$ touch each other externally. Then, by Theorem 6 , $\mathrm{f}\left(\lambda ; t_{1}\right)=0$ has a negative double root in $\lambda$. Therefore, $\Delta\left(t_{1}\right)=$ 0 . But this contradicts the condition that $\Delta(t)$ has no zero in $[0,1]$. Hence, $\mathcal{A}(t)$ and $\mathcal{B}(t)$ are collision-free.

Corollary 9: Let $\mathcal{A}(t)$ and $\mathcal{B}(t), t \in[0,1]$ be two moving elliptic disks. Suppose that $\mathcal{A}(0)$ and $\mathcal{B}(0)$ are separate. If $\Delta(t)$ has a real zero in $[0,1]$, then $\mathcal{A}(t)$ and $\mathcal{B}(t)$ touch each other externally at $t_{\min } \in[0,1]$, where $t_{\min }$ is the smallest real zero of $\Delta(t)$ in $[0,1]$, i.e., $t_{\min }=\min \{t \mid \Delta(t)=0, t \in[0,1]\}$.

The proof of Corollary 9 is similar to the proof of necessity in Theorem 8, and is therefore omitted. Here $t_{\min }$ gives the time of first contact between the disks $\mathcal{A}(t)$ and $\mathcal{B}(t)$.

\section{OUTLINE OF ALGORITHM}

Based on the separation conditions proved in the preceding sections, in this section, we shall outline the framework of our algorithm for collision detection between two moving ellipses.

\section{Algorithm: CD-DISC \\ Input: $\quad$ The matrices $A(t)$ and $B(t)$ of two moving elliptic disks $\mathcal{A}(t)$ and $\mathcal{B}(t)$. \\ Output: Whether the two elliptic disks collide: COLLISION Or COLLISION-FREE.}

Step 1: Compute the characteristic equation $\mathrm{f}(\lambda ; 0)=0$ for $\mathcal{A}(0)$ and $\mathcal{B}(0)$. Then determine whether $\mathrm{f}(\lambda ; 0)=0$ has two distinct negative roots. If yes, by Theorem 6 , $\mathcal{A}(0)$ and $\mathcal{B}(0)$ are separate, and go to Step 2; otherwise, report COLLISION and exit.

Step 2: Compute the characteristic polynomial

$$
\mathrm{f}(\lambda ; t)=\operatorname{det}(\lambda A(t)-B(t)) .
$$

Step 3: Compute the discriminant $\Delta(t)$ of $\mathrm{f}(\lambda ; t)$ with respect to $\lambda$.

Step 4: Determine whether $\Delta(t)=0$ has any real root in $[0,1]$. If yes, by Theorem 8 , report COLLISION and exit; otherwise, again by Theorem 8 , report COLLISION-FREE and exit.

In Step 1 of the algorithm CD-DISC, we use the Sturm sequence method $[19$, p. 96], a classical real-root isolation method, to check whether or not $\mathrm{f}(\lambda ; 0)=0$ has two distinct negative roots. By Theorem 6 , this can determine whether or not the two ellipses are separate at $t=0$. The Sturm sequence method counts the number of real zeros of a polynomial within a specified interval by taking the difference between the numbers of sign changes exhibited by the Sturm sequence of the polynomial at the two ends of the interval (a multiple real root is counted once only). When applying the Sturm sequence method to $f(\lambda ; 0)$ over the interval $(-\infty, 0)$, Lemma 1 ensures that the number of zeros can only be 0,1 , or 2 , corresponding to situations in which $f(\lambda ; 0)=0$ has no negative root, one negative double root, or two distinct negative roots, respectively.

The algorithm CD-DISC only reports whether the two moving ellipses collide. By solving for the roots of $\Delta(t)$, this algorithm can be extended to report also the time of first contact, or all instants at which the ellipses are in external contact. By Corollary 9 , the smallest root $t_{\min }$ of $\Delta(t)=0$ in $[0,1]$ is always the instant of first contact between the two disks. However, to report all contact instants, the other roots of $\Delta(t)=0$ in $[0,1]$ need to be checked, because while all contact instants must be roots of $\Delta(t)=0$, a root of $\Delta(t)=0$ may not correspond to an external contact between the disks. We will discuss this more in Section VI-B. For each external contact time instant $t_{i}$, the corresponding touching point of the two ellipses can be obtained by finding the unique solution to $\left(\lambda_{i} A\left(t_{i}\right)-B\left(t_{i}\right)\right) X=0$, where $\lambda_{i}$ is the negative double root of $\mathrm{f}\left(\lambda ; t_{i}\right)=0$ (Lemma 5). Implementation of the algorithm CD-DISC, with various enhancements for different types of motions and outputs, will be discussed in the following sections.

\section{NONRATIONAL Motions}

In the algorithm CD-DISC, it is necessary to at least to detect the real roots of the univariate equation $\Delta(t)=0$. When the motions of the ellipses are analytical, but otherwise arbitrary, $\Delta(t)=0$ is a rather general equation, and appropriate root-finding techniques need to be used. If the motions are piecewise analytical, then the algorithm can be applied to each piece. In the rest of the discussion, we shall consider some special types of motion that are frequently encountered, and which allow relatively easy formulation or efficient handling. In particular, we will consider the cycloidal motion in this section, and the rational motion in the next section.

The cycloidal motion is commonly used in cam design. An object with a cycloidal motion has the trajectories of all of its points being cycloids. The simplest cycloidal motion is a circle rolling along a straight line. We shall consider ellipses making cycloidal motions, such that they translate with constant velocities and, at the same time, rotate about their centers with a constant angular velocity. In this case, the elements of the motion matrix $M(t)$ contain not only rational functions of time $t$, but also trigonometric terms such as $\cos \left(\alpha_{0} t+\beta_{0}\right)$ and $\sin \left(\alpha_{1} t+\beta_{1}\right)$, for some constants $\alpha_{0}, \alpha_{1}, \beta_{0}, \beta_{1}$. Therefore, the coefficients $\mathrm{g}_{i}(t)$ of the characteristic polynomial in (1) are not rational functions in $t$. It might be suggested that the trigonometric functions could be converted intorational functions using the variable substitution $u=\tan (t / 2)$. However, this substitution would make the translational part, which is linear in $t$, nonrational. In fact, this kind of motion is intrinsically transcendental; hence, it can only be approximated, but not exactly represented, by a rational motion.

Suppose that two elliptic disks $\mathcal{A}$ and $\mathcal{B}$ perform cycloidal motions. Since the sizes and shapes of the disks do not change during the motion, the coefficients $g_{3}(t)$ and $g_{0}(t)$ of (1) are constant and equal to $\operatorname{det}(A)$ and $-\operatorname{det}(B)$, respectively. Let $u=(1-t) \theta_{0}+t \theta_{1}$ and $v=(1-t) \phi_{0}+t \phi_{1}$ be linear interpolations of the initial and final orientation angles $\theta_{0}, \theta_{1}$ of $\mathcal{A}$, and $\phi_{0}, \phi_{1}$ of $\mathcal{B}$. Then the other two coefficients, $\mathrm{g}_{2}(t)$ and 
$\mathrm{g}_{1}(t)$, can be expressed as

$$
\begin{aligned}
\mathrm{g}_{2}(t)= & \left(\alpha_{22} \cos (2 v)+\alpha_{21} \sin (2 v)+\alpha_{20}\right) t^{2} \\
& +\left(\alpha_{12} \cos (2 v)+\alpha_{11} \sin (2 v)+\alpha_{10}\right) t \\
& +\alpha_{03} \cos (2(u-v))+\alpha_{02} \cos (2 v) \\
& +\alpha_{01} \sin (2 v)+\alpha_{00} \\
\mathrm{~g}_{1}(t)= & \left(\beta_{22} \cos (2 u)+\beta_{21} \sin (2 u)+\beta_{20}\right) t^{2} \\
& +\left(\beta_{12} \cos (2 u)+\beta_{11} \sin (2 u)+\beta_{10}\right) t \\
& +\beta_{03} \cos (2(u-v))+\beta_{02} \cos (2 u) \\
& +\beta_{01} \sin (2 u)+\beta_{00}
\end{aligned}
$$

where the $\alpha$ and $\beta$ terms are all constants.

Since the coefficients $\mathrm{g}_{i}(t)$ are not rational, neither is the discriminant $\Delta(t)$ for a cycloidal motion. One may use any suitable numerical solver to compute the roots of $\Delta(t)=0$ or to check for the existence of any real roots. The example below illustrates the steps of the algorithm CD-DISC for two ellipses making cycloidal motions.

Example 1: Consider two elliptic disks $\mathcal{A}: x^{2} / 6^{2}+y^{2} / 10^{2}$ $\leq 1$ and $\mathcal{B}: x^{2} / 14^{2}+y^{2} / 4^{2} \leq 1$. Two moving elliptic disks, $\mathcal{A}(t)$ and $\mathcal{B}(t), t \in[0,1]$, are defined by the transformation of $\mathcal{A}$ and $\mathcal{B}$ under the following cycloidal motions:

$$
\begin{aligned}
M_{A} & =\left(\begin{array}{ccc}
\cos \left(\frac{10 \pi t}{9}\right) & -\sin \left(\frac{10 \pi t}{9}\right) & 115 t-80 \\
\sin \left(\frac{10 \pi t}{9}\right) & \cos \left(\frac{10 \pi t}{9}\right) & 55 t-38 \\
0 & 0 & 1
\end{array}\right) \\
M_{B} & =\left(\begin{array}{ccc}
\cos \left(\frac{2 \pi t}{3}\right) & -\sin \left(\frac{2 \pi t}{3}\right) & 76 t-60 \\
\sin \left(\frac{2 \pi t}{3}\right) & \cos \left(\frac{2 \pi t}{3}\right) & 97 t-57 \\
0 & 0 & 1
\end{array}\right) .
\end{aligned}
$$

The characteristic polynomial is

$$
\begin{aligned}
\mathrm{f}(\lambda ; t)= & \operatorname{det}(\lambda A(t)-B(t)) \\
= & -\frac{1}{3600} \lambda^{3}+\left(\left(-\frac{243}{125440} \cos \left(\frac{4 \pi t}{3}\right)\right.\right. \\
& \left.-\frac{117}{4480} \sin \left(\frac{4 \pi t}{3}\right)-\frac{3869}{125440}\right) t^{2} \\
& +\left(\frac{9}{31360} \cos \left(\frac{4 \pi t}{2}\right)+\frac{1581}{62720} \sin \left(\frac{4 \pi t}{3}\right)\right. \\
& \left.+\frac{13939}{470400}\right) t+\frac{1}{1960} \cos \left(\frac{8 \pi t}{9}\right) \\
& +\frac{39}{125440} \cos \left(\frac{4 \pi t}{3}\right)-\frac{19}{3136} \\
& \left.\times \sin \left(\frac{4 \pi t}{3}\right)-\frac{10519}{1881600}\right) \lambda^{2} \\
& +\left(\left(-\frac{27}{39200} \cos \left(\frac{20 \pi t}{9}\right)-\frac{13}{1400}\right.\right. \\
& \left.\times \sin \left(\frac{20 \pi t}{9}\right)+\frac{1241}{62720}\right) t^{2} \\
& +\left(\frac{1}{9800} \cos \left(\frac{20 \pi t}{9}\right)\right. \\
& \left.+\frac{527}{58800} \sin \left(\frac{20 \pi t}{9}\right)-\frac{4471}{235200}\right) t \\
& -\frac{1}{1960} \cos \left(\frac{8 \pi t}{9}\right)+\frac{13}{117600} \cos \left(\frac{20 \pi t}{9}\right) \\
& \left.-\frac{19}{8820} \sin \left(\frac{20 \pi t}{9}\right)+\frac{937}{313600}\right) \lambda+\frac{1}{3136} .
\end{aligned}
$$

The discriminant $\Delta(t)$ has a long expression, and is therefore omitted.

The disks $\mathcal{A}(t)$ and $\mathcal{B}(t)$, moving from left to right, and the graph of their discriminant are shown in Fig. 5. Using Maple, with floating-point computations to 12 decimal places, the roots are found at $t=0.226,0.393,0.600,0.731$. Therefore, the two ellipses collide during the cycloidal motion, the first contact is at time $t=0.226$, and the contact point is found at $(-47.605,-33.162)^{T}$. Furthermore, only $t=0.731$ corresponds to another external touching of the two ellipses, and the

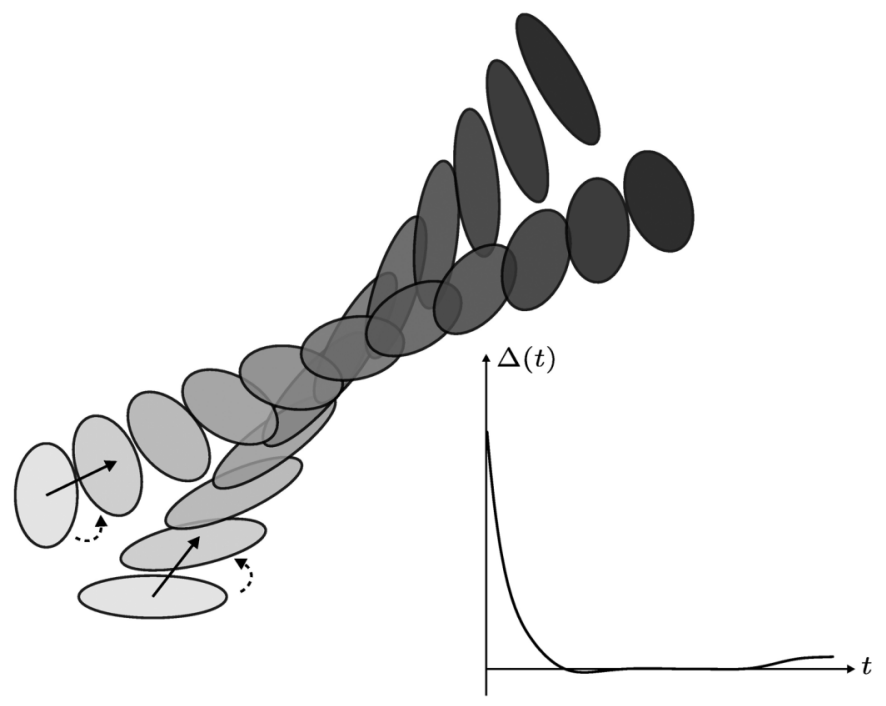

Fig. 5. Two moving ellipses of Example 1, progressing from left to right, and the discriminant $\Delta(t)$.

contact point is $(-2.469,2.723)^{T}$. Comparing with the results obtained by Maple integer arithmetics (except for the last step of root solving using floating-point computations), the accuracy of the roots found above is up to nine decimal places.

\section{RATIONAL MOTIONS}

Recent studies on rational motions [20]-[23], and in particular, on planar rational motions [24], have shown that low-degree rational motions are adequate to meet the need for motion design and representation in robotics and CAD/CAM. The use of rational motions also allows effective computation, using various well-developed techniques for processing polynomials. In this section, we shall study in detail the application of our method to collision detection between two elliptic disks making rational motions, with particular emphasis on rational Euclidean motions. The resulting algorithms are also applicable to affine motions that produce continuous deformation of the objects, an effect often required in computer animation.

\section{A. Planar Rational Euclidean Motions}

We start with a brief review of planar rational Euclidean motions. A Euclidean transformation in $\mathbb{E}^{2}$ is given by $X^{\prime}=M X$, where

$$
M=\rho\left(\begin{array}{cc}
R & V \\
0^{T} & 1
\end{array}\right)
$$

for some nonzero constant $\rho$, and $X, X^{\prime}$ are points in $\mathbb{E}^{2}$ in homogeneous coordinates. The rotational part of the transformation is described by the $2 \times 2$ orthogonal matrix $R$, and the translational part by the vector $V$. If the elements of $R$ and $V$ are continuous functions of $t$, then $M$ describes a transformation over time, and can, therefore, be denoted by $M(t)$. In particular, if the elements of $M(t)$ are rational functions and $R$ is orthogonal for all $t$, then $M(t)$ is called a rational Euclidean motion whose degree is the maximal degree of its elements. (Note that $M(t)$ represents an affine motion only if $R(t)$ is nonsingular.) 
One way to construct a rational Euclidean motion is to use the kinematic mapping that associates the Euclidean transformation $M$ with a point $\mathbf{d}$ in $\mathbb{P}^{3}$, the 3-D real projective space, as described in [24]. If we write

$$
R=\left(\begin{array}{cc}
\cos \phi & -\sin \phi \\
\sin \phi & \cos \phi
\end{array}\right) \text { and } V=\left(\begin{array}{l}
v_{x} \\
v_{y}
\end{array}\right)
$$

then the kinematic image $\mathbf{d} \in \mathbb{P}^{3}$ of $M$ is given by

$$
\mathbf{d}=\left(\begin{array}{l}
d_{0} \\
d_{1} \\
d_{2} \\
d_{3}
\end{array}\right)=\left(\begin{array}{c}
v_{x} \sin (\phi / 2)-v_{y} \cos (\phi / 2) \\
v_{x} \cos (\phi / 2)+v_{y} \sin (\phi / 2) \\
-2 \cos (\phi / 2) \\
2 \sin (\phi / 2)
\end{array}\right) .
$$

Conversely, any point $\mathbf{d}$ in $\mathbb{P}^{3}$ at which $d_{2}^{2}+d_{3}^{2} \neq 0$ corresponds to a Euclidean transformation $M$ in $\mathbb{E}^{2}$, given by

$$
M=\left(\begin{array}{ccc}
d_{2}^{2}-d_{3}^{2} & 2 d_{2} d_{3} & 2\left(d_{0} d_{3}-d_{1} d_{2}\right) \\
-2 d_{2} d_{3} & d_{2}^{2}-d_{3}^{2} & 2\left(d_{0} d_{2}+d_{1} d_{3}\right) \\
0 & 0 & d_{2}^{2}+d_{3}^{2}
\end{array}\right)
$$

It follows that there is a one-to-one correspondence between a Euclidean transformation in $\mathbb{E}^{2}$ and a point in the kinematic image space, which is $\mathbb{P}^{3}$ with the line $d_{2}=d_{3}=0$ removed. Due to this correspondence by means of a kinematic mapping, we may construct a polynomial curve in the kinematic image space and then obtain the corresponding rational Euclidean motion in $\mathbb{E}^{2}$. In general, if the $d_{i}$ terms are polynomials of degree $n$, the resulting motion will be of degree $2 n$. A $C^{2}$ interpolation scheme of a set of given positions in $\mathbb{E}^{2}$ with piecewise quartic B-spline rational motions can be found [24]. Another advantage of rational motions is that they permit an algebraic treatment of the collision-detection problem.

When applying a rational motion $M(t)$ to an ellipse $\mathcal{A}$ : $X^{T} A X=0$, we get a moving ellipse $\mathcal{A}(t): X^{T} A(t) X=0$, where

$$
A(t)=\left(M^{-1}(t)\right)^{T} A M^{-1}(t) .
$$

Inverting (3), we have

$$
M^{-1}=\left(\begin{array}{ccc}
d_{2}^{2}-d_{3}^{2} & -2 d_{2} d_{3} & 2\left(d_{1} d_{2}+d_{0} d_{3}\right) \\
2 d_{2} d_{3} & d_{2}^{2}-d_{3}^{2} & 2\left(d_{1} d_{3}-d_{0} d_{2}\right) \\
0 & 0 & d_{2}^{2}+d_{3}^{2}
\end{array}\right) .
$$

Therefore, the maximal degree of the entries in $A(t)$ is $2 k$, if the degree of the motion $M(t)$ is $k$.

\section{B. Properties of $\Delta(t)$}

We now analyze the degree of the discriminant $\Delta(t)$. The characteristic equation $\mathrm{f}(\lambda ; t)=0$ of the two moving elliptic disks $\mathcal{A}(t)$ and $\mathcal{B}(t)$ is cubic in $\lambda$, and its degree in $t$ depends on the degree of the rational motions of the two disks. Suppose that the motions $M_{A}(t)$ and $M_{B}(t)$ both have degree $k$. Then the maximum degree of the elements of $\lambda A(t)-B(t)$ is $2 k$, and the maximum degree of the coefficients $g_{i}(t)$ of the characteristic equation is $6 k$. Hence, from (2), the maximum degree of $\Delta(t)$ is $24 k$. This analysis only gives an upper bound of the degree of $\Delta(t)$, because the actual degree of $\Delta(t)$ depends on the specific motions that are used. For example, consider a linear translational motion

$$
M(t)=\left(\begin{array}{cc}
R(t) & V(t) \\
\mathbf{0}^{T} & 1
\end{array}\right)
$$

TABLE I

DEgREES OF VARIOUS ENTITIES FOR RATIONAL MOTIONS OF DIFFERENT DEGREeS. THE LAST Row SHOWS MAXIMUM DEgREES OF THE ENTITIES FOR A General Motion of Degree $k$. The Motion $M(t)$ TAKes the Form of (3)

\begin{tabular}{c||c|c|c}
\hline \multirow{2}{*}{\multicolumn{1}{c||}{}} & \multicolumn{3}{c}{ Degree in $t$} \\
\cline { 2 - 4 } Motion Type & $M(t)$ & $\mathrm{g}_{i}(t)$ & $\Delta(t)$ \\
\hline \hline Linear Translation & 1 & $0\left(\mathrm{~g}_{0}, \mathrm{~g}_{3}\right), 2\left(\mathrm{~g}_{1}, \mathrm{~g}_{2}\right)$ & 8 \\
\hline General Motion & $k$ & $6 k$ & $24 k$ \\
\hline
\end{tabular}
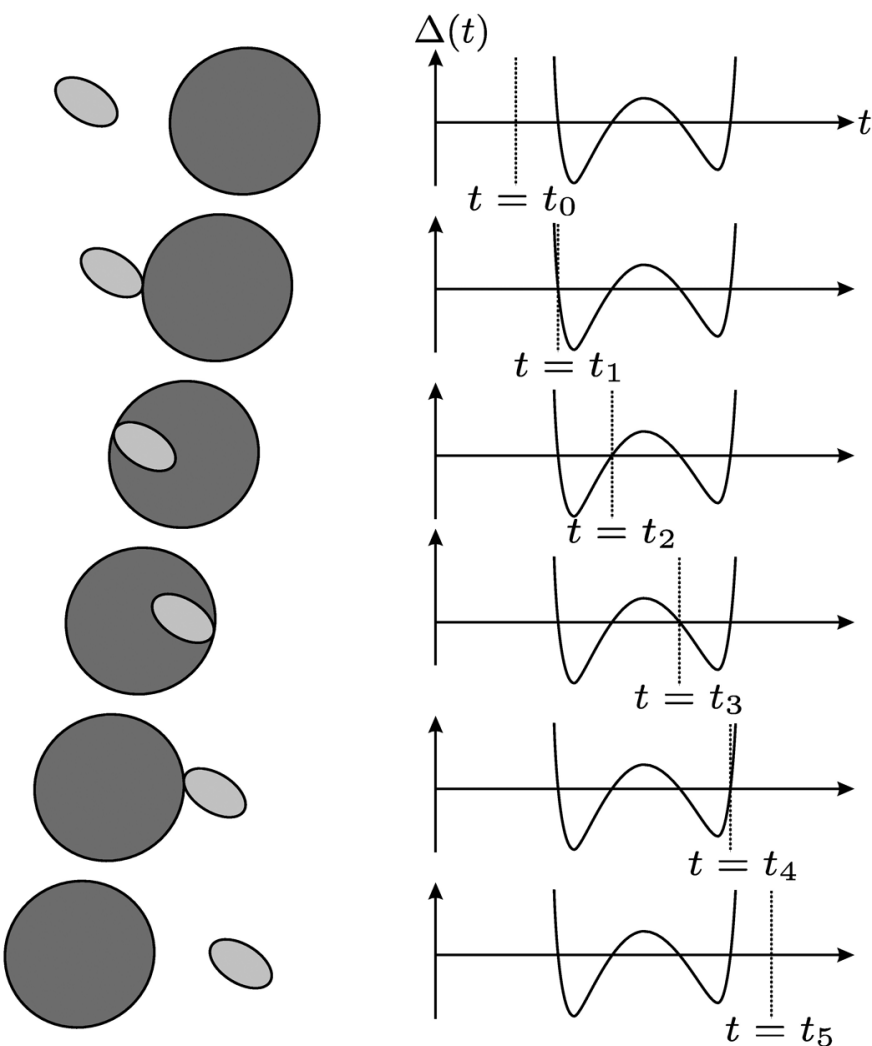

Fig. 6. Two elliptic disks with translational motions, and their corresponding discriminant function.

where the entries in $R(t)$ are all constants, and those in $V(t)$ are linear polynomials. Then, the degree of $\mathrm{g}_{0}$ and $\mathrm{g}_{3}$ is 0 , while that of $g_{1}$ and $g_{2}$ is 2 . Therefore, the degree of $\Delta(t)$ is only 8 , which is much lower than 24 , which is the degree that could be deduced from the general analysis. The relationship between the degree of $\Delta(t)$ and the degree of the rational motions is summarized in Table I.

Now let us consider the geometric meaning of the roots of $\Delta(t)$. If two ellipses $\mathcal{A}\left(t_{0}\right)$ and $\mathcal{B}\left(t_{0}\right)$ touch each other externally, by Theorem $6, \mathrm{f}\left(\lambda, t_{0}\right)=0$ has a negative double root, and we have $\Delta\left(t_{0}\right)=0$. However, when $\Delta(\tilde{t})=0$ for some $\tilde{t}$, the disks $\mathcal{A}(\tilde{t})$ and $\mathcal{B}(\tilde{t})$ do not necessarily touch each other.

Fig. 6 shows two elliptic disks moving with linear translational motions and the graph of their discriminant. Here, $\mathcal{A}(0)$ and $\mathcal{B}(0)$ are separate. Note that the first real root $t_{1}$ of $\Delta(t)=0$ corresponds to an external contact between $\mathcal{A}(t)$ and $\mathcal{B}(t)$, while the next two roots, $t_{2}$ and $t_{3}$, are caused by internal tangency of the two disks.

Since the degree of $\Delta(t)$ is eight in the case of a linear translational motion, $\Delta(t)=0$ can have eight real roots at most. Fig. 7 illustrates a case where all eight real roots are accounted for by real tangencies between two elliptic disks during linear 


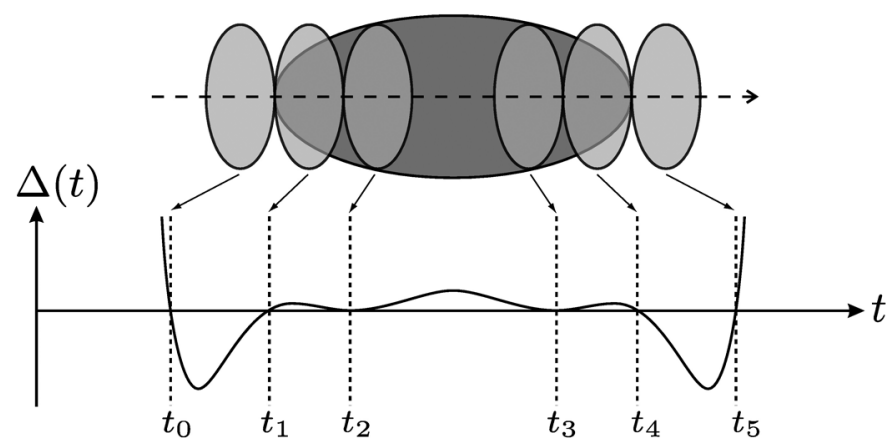

Fig. 7. Eight real roots of $\Delta(t)=0$ for a translational motion, and the corresponding contact points between the two elliptic disks. Note the double roots at $t_{2}$ and $t_{3}$.

motions. Here, there are two instants $\left(t_{2}\right.$ and $\left.t_{3}\right)$ when the two disks are internally tangential to each other simultaneously at two points; $t_{2}$ and $t_{3}$ are double zeros of $\Delta(t)=0$.

We have mentioned that a real zero of $\Delta(t)=0$ may not correspond to any real tangency between the two ellipses. To see this, consider two moving circular disks that become two concentric circles $x^{2}+y^{2}=1$ and $x^{2}+y^{2}=4$ at time $t_{0}$. It is easy to verify that the characteristic equation $\mathrm{f}\left(\lambda ; t_{0}\right)=0$ has a positive double root, and therefore, $\Delta\left(t_{0}\right)=0$. But the two circles have no real touching point: the two circles are tangential to each other at two complex conjugate points $(1, \pm i, 0)$, known as the circular points in projective geometry. This explains why only the first real root of $\Delta(t)$ in $t \in[0,1]$ always indicates an external contact between two moving elliptic disks, as assured by Corollary 9 , on condition that the two elliptic disks are separate at the beginning (i.e., when $t=0)$. For each of the other real roots $\bar{t}$ of $\Delta(t)=0$, we need to check the root pattern of the characteristic polynomial at time $\bar{t}$ to see whether there is an external contact. Theorem 61 ) tells us that a root $\bar{t}$ of $\Delta(t)=0$ corresponds to an external tangency of the two ellipses iff the characteristic polynomial $\mathrm{f}(\lambda ; \bar{t})=0$ has a negative double root in $\lambda$.

\section{Robust Computation}

In this section, we shall discuss robust implementation of CD-DISC for testing collisions between two elliptic disks moving with rational motions. One of the steps in CD-DISC (see Section IV) is to construct the discriminant $\Delta(t)$. The discriminant is a univariate polynomial in $t$ that is the result of long polynomial computations (mainly polynomial multiplications) from the coefficients of the motion matrices, which are also polynomials. If the computations are carried out using the power series representation of the polynomials (i.e., $\left.\mathrm{p}(t)=\sum_{i=0}^{n} a_{i} t^{i}, a_{i} \in \mathbb{R}\right)$, we found that CD-DISC suffered severely from numerical instability when the degree of motion is higher than two, using double-precision floating-point arithmetic. By comparing intermediate results through the entire process with exact results produced by a Maple implementation of the same algorithm using exact integer computations, significant errors in the coefficients of $\Delta(t)$ are revealed. We perform a test, in which two elliptic disks move with degree-4 motion, using Maple with high-precision floating-point computation, and found that acceptable results could only be obtained when the number of decimal places in the floating-point computation is increased above 20. In this case, the degree of $\Delta(t)$ is 96 .
To overcome this numerical instability in processing high-degree polynomials, we turned to the Bernstein form of polynomials. The Bernstein form has the expression

$$
\sum_{i=0}^{n}\left(\begin{array}{c}
n \\
i
\end{array}\right) a_{i} t^{i}(1-t)^{n-i}, a_{i} \in \mathbb{R}
$$

and is known to be numerically more stable for polynomial computations than the power form [25], [26]. In our current implementation of CD-DISC, we still use polynomials in the power form when computing the characteristic equation $\mathrm{f}(\lambda ; t)=0$ from the motion matrices, and then convert the coefficients $g_{i}(t)$ of $\mathrm{f}(\lambda ; t)=0$ into the Bernstein form; the numerical condition of this conversion is satisfactory, since the terms $g_{i}(t)$ have relatively low degrees [27]. Finally, we derive $\Delta(t)$ by computing with polynomials in the Bernstein form. Our experiments show that this adoption of the Bernstein form significantly improves the robustness and accuracy of our collision-detection procedure.

Having obtained the discriminant $\Delta(t)$ in a robust manner, the next step is to analyze its zeros. The extent of processing of $\Delta(t)$ depends on what kind of collision-detection output is required by an application. The following three variants of CD-DISC have been implemented that give different collision-detection outputs for two elliptic disks making rational motions.

Variant 1) reports whether the two elliptic disks collide.

Variant 2) reports whether the two elliptic disks collide, and, if so, reports the time of first contact.

Variant 3) reports whether the two elliptic disks collide, and, if so, reports all instants of external contact.

These different output types require different ways of handling the discriminant $\Delta(t)=0$. For Variant 1 , we only need to check for the existence of real roots of $\Delta(t)=0$. Here we make use of a recent result that uses an idea similar to the Sturm sequence method to count the number of real roots of a polynomial in the scaled Bernstein form [28], which is expressed as $\sum_{i=0}^{n} b_{i} t^{i}(1-t)^{n-i}$, where $b_{i} \in \mathbb{R}$. This method inherits the robustness provided by the Bernstein form, and therefore, is suitable for high-degree polynomials. Using this technique, we are able to determine robustly whether $\Delta(t)$ has any real roots in the time interval $t \in[0,1]$. Here, the coefficients $g_{i}(t)$ of the characteristic equation $\mathrm{f}(\lambda ; t)=0$ are first transformed to the scaled Bernstein form, and then $\Delta(t)$ is obtained by computing with polynomials in the scaled Bernstein form. This treatment avoids the errors that would otherwise be caused by the high degree of $\Delta(t)$, if $\Delta(t)$ were first obtained in the Bernstein basis and then transformed into the scaled Bernstein form. The scaled Bernstein form is used only in Variant 1.

For Variant 2, we need to solve for the smallest real root of $\Delta(t)=0$ in $[0,1]$, if one exists. For Variant 3 , we must obtain all the real roots of $\Delta(t)=0$ in $[0,1]$. For each of these roots, we also need to check for the existence of a negative double root of the characteristic polynomial, in order to verify the external tangency of the two elliptic disks. In both Variants 2 and 3, we use the de Casteljau algorithm to subdivide $\Delta(t)$ in the Bernstein form to locate all real roots of $\Delta(t)$ in $[0,1]$. Using the convex hull property, we can discard an interval of $t$ if the Bernstein coefficients of $\Delta(t)$ over that interval are all positive or 


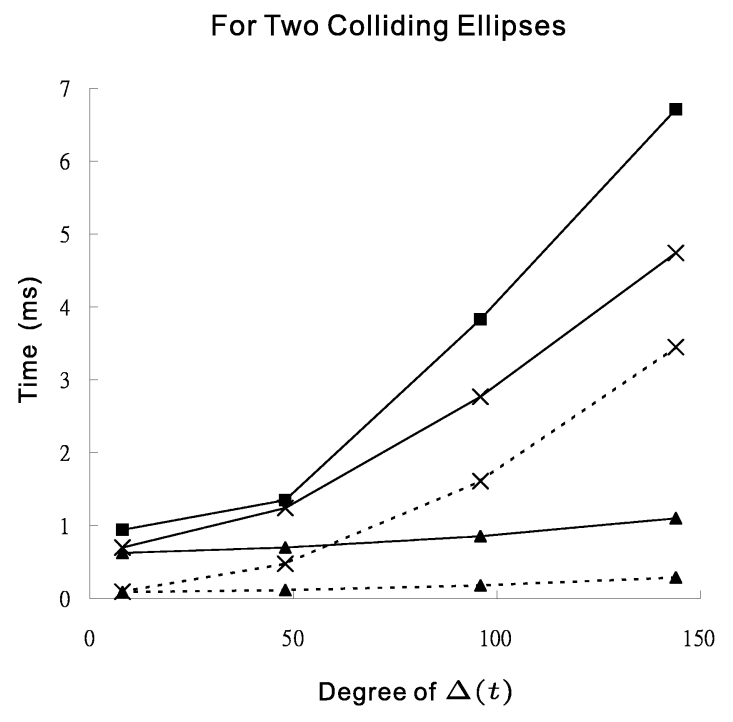

(a)

(1). Average

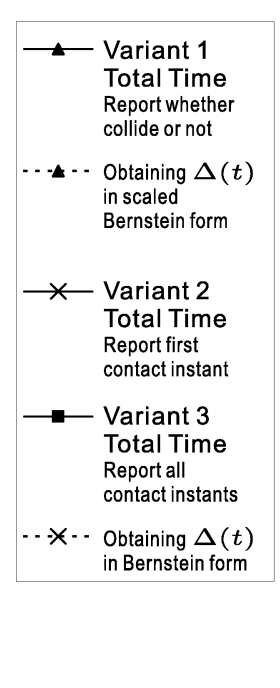

Fig. 8. Average CPU time needed for CD-DISC to detect collision for two moving elliptic disks with different degrees of motion, when the two moving elliptic disks (a) collide, or (b) are collision-free. The solid lines show the total collision detection time for the three variants of CD-DISC (see Section VI-C), and the dashed lines show the computation time taken for obtaining $\Delta(t)$ in the Bernstein form and in the scaled Bernstein form.

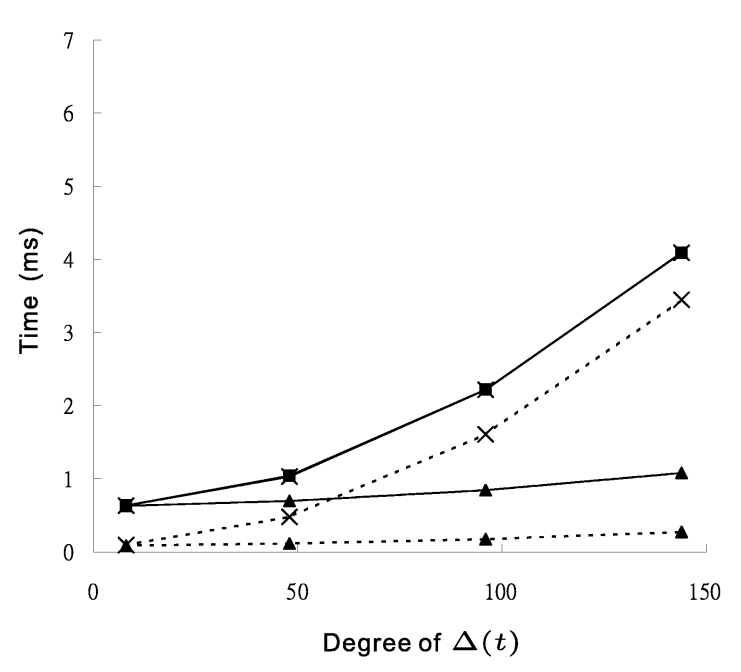

(b) all negative. For Variant 2, in which only the first contact-time instant is needed, we can save computation time by continuing to subdivide only those intervals within which the smallest real root might be contained.

\section{Experimental Results}

We shall first use a large set of synthesized motions to demonstrate the efficiency of our collision-detection algorithm, and then use a more detailed example to show its accuracy. We generated 2000 test cases for each of four kinds of motion: linear translations and general rational motions of degrees 2,4 , and 6 . In each set of 2000 cases, 1000 cases were randomly generated pairs of colliding elliptic disks, and the other 1000 were randomly generated pairs of collision-free elliptic disks. The experiments were run on a PC with a $2.2 \mathrm{GHz}$ Intel CPU, and the timings are shown in Fig. 8. The graphs in Fig. 8(a) and (b) give the average CPU time taken by CD-DISC for colliding and collision-free elliptic disks, respectively. The three solid lines correspond to the three different outputs (Variants 1-3, as described in Section VI-C) that CD-DISC can report. Clearly, more time is needed as the degree of motion, and hence, the degree of $\Delta(t)$ increases. For two elliptic disks making a motion of degree 6 , for which the degree of $\Delta(t)$ is 144 , it takes less than $1 \mathrm{~ms}$ to determine whether there is any collision, less than $5 \mathrm{~ms}$ to compute the instant of first contact, and less than $7 \mathrm{~ms}$ to compute all instants of contact.

For both colliding and collision-free ellipses, Variant 1 of the algorithm takes the same time, since the computation involved to decide whether there is a collision (i.e., to determine root existence by Sturm sequences) is the same in both cases. In the case of colliding elliptic disks [Fig. 8(a)], more time is needed for Variants 2 and 3 to detect the instants of contact; in general, the computation time increases as the number of roots of $\Delta(t)$ increases. When there is no collision [Fig. 8(b)], $\Delta(t)$ has no root, and the average CPU time taken for reporting the instant of first contact (Variant 2) or the instants of all contacts (Variant 3 ) is the same.

The dashed lines in the graphs show the time needed for obtaining the polynomial $\Delta(t)$ from the characteristic equation; in Variant $1, \Delta(t)$ is obtained in the scaled Bernstein form, while in Variants 2 and 3, the computation is done in the Bernstein form. It is obvious that in Variants 2 and 3, obtaining $\Delta(t)$ takes up most of the overall time for collision detection. Polynomial multiplications in the scaled Bernstein form are much more efficient than those in the Bernstein form, which explains why the time needed for obtaining $\Delta(t)$ in Variant 1 is much less than that needed in Variants 2 and 3.

Next, we use a worked example to show the robustness of the algorithm CD-DISC.

Example 2: Consider two ellipses $\mathcal{A}: x^{2} / 5^{2}+y^{2} / 10^{2}=1$ and $\mathcal{B}: x^{2} / 5^{2}+y^{2} / 10^{2}=1$. Two moving elliptic disks $\mathcal{A}(t)$ and $\mathcal{B}(t), t \in[0,1]$, are defined by applying to $\mathcal{A}$ and $\mathcal{B}$ the following motions $M_{A}$ and $M_{B}$ :

$$
\begin{aligned}
& M_{A}= \\
& \left(\begin{array}{ccc}
-16 t^{4}+32 t^{3} & -32 t^{3}+48 t^{2} & -160 t^{3}-240 t^{2} \\
-16 t+4 & -16 t & +160 t-40 \\
32 t^{3}-48 t^{2} & -16 t^{4}+32 t^{3} & 480 t^{4}-960 t^{3} \\
+16 t & -16 t+4 & +880 t^{2}-400 t+80 \\
0 & 0 & 16 t^{4}-32 t^{3}+32 t^{2} \\
0 & & -16 t+4
\end{array}\right) \\
& \left.M_{B}=\begin{array}{ccc}
-16 t^{4}+32 t^{3} & 32 t^{3}-48 t^{2} & 160 t^{3}-240 t^{2} \\
-16 t+4 & +16 t & +160 t-40 \\
-32 t^{3}+48 t^{2} & -16 t^{4}+32 t^{3} & -480 t^{4}+960 t^{3} \\
-16 t & -16 t+4 & -880 t^{2}+400 t-80 \\
0 & 0 & 16 t^{4}-32 t^{3}+32 t^{2} \\
0 & 0 & -16 t+4
\end{array}\right) .
\end{aligned}
$$


The characteristic equation is

$$
\begin{aligned}
& \mathrm{f}(\lambda ; t) \\
& =\operatorname{det}(\lambda A(t)-B(t)) \\
& =\left(-4096 t^{24}+49152 t^{23}-294912 t^{22}\right. \\
& +1171456 t^{21}-3446784 t^{20}+7974912 t^{19} \\
& -15048704 t^{18}+23721984 t^{17} \\
& -31756032 t^{16}+36517888 t^{15}-36360192 t^{14} \\
& +31509504 t^{13}-23835904 t^{12}+15754752 t^{11} \\
& -9090048 t^{10}+4564736 t^{9}-1984752 t^{8} \\
& +741312 t^{7}-235136 t^{6}+62304 t^{5} \\
& \left.-13464 t^{4}+2288 t^{3}-288 t^{2}+24 t-1\right) \lambda^{3} \\
& +\left(-135168 t^{24}+1622016 t^{23}-11304960 t^{22}\right. \\
& +55959552 t^{21}-206878720 t^{20} \\
& +588967936 t^{19}-1325514752 t^{18} \\
& +2409461760 t^{17}-3596409600 t^{16} \\
& +4461631488 t^{15}-4639457280 t^{14}+4065807360 t^{13} \\
& -3011391744 t^{12}+1886084608 t^{11}-997282816 t^{10} \\
& +443501312 t^{9}-164884848 t^{8}+50819520 t^{7} \\
& -12842880 t^{6}+2624352 t^{5}-426168 t^{4} \\
& \left.+53808 t^{3}-5120 t^{2}+344 t-13\right) \lambda^{2} \\
& +\left(135168 t^{24}-1622016 t^{23}+11304960 t^{22}\right. \\
& -55959552 t^{21}+206878720 t^{20} \\
& -588967936 t^{19}+1325514752 t^{18} \\
& -2409461760 t^{17}+3596409600 t^{16}-4461631488 t^{15} \\
& +4639457280 t^{14}-4065807360 t^{13} \\
& +3011391744 t^{12}-1886084608 t^{11}+997282816 t^{10} \\
& -443501312 t^{9}+164884848 t^{8}-50819520 t^{7} \\
& +12842880 t^{6}-2624352 t^{5}+426168 t^{4} \\
& \left.-53808 t^{3}+5120 t^{2}-344 t+13\right) \lambda \\
& +4096 t^{24}-49152 t^{23}+294912 t^{22} \\
& -1171456 t^{21}+3446784 t^{20}-7974912 t^{19} \\
& +15048704 t^{18}-23721984 t^{17}+31756032 t^{16} \\
& -36517888 t^{15}+36360192 t^{14} \\
& -31509504 t^{13}+23835904 t^{12}-15754752 t^{11} \\
& +9090048 t^{10}-4564736 t^{9}+1984752 t^{8} \\
& -741312 t^{7}+235136 t^{6}-62304 t^{5}+13464 t^{4} \\
& -2288 t^{3}+288 t^{2}-24 t+1 \\
& =0
\end{aligned}
$$

while the discriminant $\Delta(t)$ is of degree 96 and is omitted. Fig. 9 shows the two moving elliptic disks and the graph of $\Delta(t)$. The two disks are designed only to touch each other externally at $t=0.5$, and are separate for the rest of the time. All three variants of CD-DISC determined correctly that there is a contact, and Variants 2 and 3 reported the contact time accurately as $t=0.5$. To examine the sensitivity of CD-DISC, the lower disk in Fig. 9 was translated by a small amount in the negative $y$ direction, so that the two moving disks attain a minimum separating distance $d>0$ at $t=0.5$. CD-DISC reports collision

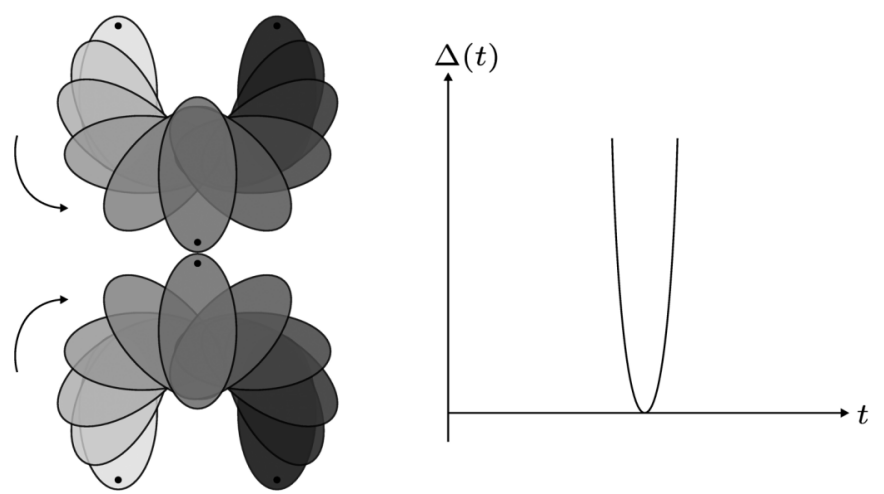

Fig. 9. Two moving elliptic disks in Example 2 and their discriminant $\Delta(t)$.

when $d<10^{-6}$, and reports noncollision for larger values of $d$. Note that the above analysis is only based on the specific setup in this example. The robustness of the method depends not only on the degree of the motion, but also varies with different input, e.g., sizes of the ellipses. However, it is not easy to formulate the theoretical condition for the robustness of this method. If an error bound is crucial to an application, one may consider the use of the interval arithmetic [29] in the computations.

\section{CONCLUSION}

We have presented a collision-detection algorithm for two elliptic disks moving with continuous motions in the plane. The algorithm, called CD-DISC, is based on an algebraic characterization of two collision-free elliptic disks, which can assume rigid or deformable motions. CD-DISC uses exact representations for the ellipses, and therefore, does not suffer from errors induced by polygonal approximations. The algorithm determines whether there is a collision by checking for the existence of real roots of a univariate function, which is the discriminant of the characteristic equation of the two moving ellipses. It may also report the time instants at which the two ellipses are externally touching, and the corresponding contact points. Unlike many other collision-detection algorithms, CD-DISC does not use temporal sampling of the motion path, so inaccuracy due to limited sampling resolution is avoided.

We have studied commonly used cycloidal motions and rational motions in detail. For motions that are intrinsically nonrational, such as cycloidal motions, the algorithm can be used with the aid of any numerical solver that will deal with a univariate function. Rational motions are also considered because they are flexible enough for modeling general motions, and their polynomial representation makes an algebraic treatment of collision detection possible: in this case, collision detection is achieved by detecting or finding the real roots of a univariate polynomial. We have demonstrated that the use of Bernstein forms for polynomial manipulation significantly increases numerical stability of CD-DISC for high-degree rational motions, which conforms to others' observations [25]-[27]. Our experiments show that CD-DISC is fast and accurate for detecting collisions between moving ellipses under continuous rational motions of degree six or less; note that research [24] in planar rational motions suggests that rational motions of degree four are adequate for modeling all smooth motions in practice. 
There are several problems open for further research. Knowing the minimum distance between two collision-free moving elliptic disks would be useful for motion path planning. Work is needed to study the relationship between the minimum distance and the value of the discriminant $\Delta(t)$. An observation that can already be made is that the difference between the two negative roots of the characteristic polynomial becomes smaller as the two separate disks approach each other gradually, and eventually the two roots merge into a negative double root, at which point the two disks become externally tangential to each other, signaled by the vanishing of the discriminant function.

Devising an algebraic approach to CCD of moving ellipsoids in 3-D space is another interesting but challenging problem. Unfortunately, it would not be a straightforward task to extend CD-DISC directly to moving ellipsoids. It has been proved [16] that the quartic characteristic equation $\mathrm{f}(\lambda) \equiv \operatorname{det}(\lambda A-B)=0$ of two ellipsoids in 3-D always has two positive roots, and two ellipsoids are separate iff $\mathrm{f}(\lambda)=0$ has two distinct negative roots. Unlike the situation with elliptic disks (see Corollary 7), in 3-D the characteristic equation $\mathrm{f}(\lambda)=0$ may have a positive double root for a pair of separate ellipsoids (compare the characteristic equation of two separate spheres, which always has a double root $\lambda=1$ ). Thus, if we were to rely on detecting real zeros of the discriminant for collision detection between moving ellipsoids, our algorithm would certainly fail (i.e., have a false positive) because a zero of the discriminant can be caused by a positive double root, which does not correspond to an external contact between the ellipsoids. To circumvent this difficulty, a method has been proposed [17] for CCD between ellipsoids by analyzing the zero-set topology of the bivariate function $\mathrm{f}(\lambda ; t)=0$. The main issue with this method is how to extract the contact instants of two moving ellipsoids by parsing the zero-set of a high-degree bivariate polynomial resulting from general rational motions in an efficient way.

\section{REFERENCES}

[1] J. Canny, "Collision detection for moving polyhedra," IEEE Trans. Pattern Anal. Mach. Intell., vol. PAMI-8, no. 2, pp. 200-209, Mar. 1986.

[2] P. Jiménez, F. Thomas, and C. Torras, "3D collision detection: A survey," Comput. Graph., vol. 25, no. 2, pp. 269-285, 2001.

[3] C. Ó. Dúnlaing and C. K. Yap, "A retraction method for planning the motion of a disc," J. Algorithms, vol. 6, pp. 104-111, 1985.

[4] F. P. Preparata and M. I. Shamos, Computational Geometry: An Introduction. New York: Springer-Verlag, 1985.

[5] M. I. Shamos and D. Hoey, "Geometric intersection problems," in Proc. 17th Annu. IEEE Symp. Found. Comput. Sci., 1976, pp. 208-215.

[6] M. Sharir, "Intersection and closest-pair problems for a set of planar discs," SIAM J. Comput., vol. 14, no. 2, pp. 448-468, 1985.

[7] M. Berger, Geometry. Berlin, Germany: Springer-Verlag, 1987, vol. II.

[8] T. J. I. Bromwich, Quadratic Forms and Their Classification by Means of Invariant-Factors, ser. Cambridge Tracts in Mathematics and Mathematical Physics. New York: Hafner, 1906, vol. 3.

[9] J. G. Semple and G. Kneebone, Algebraic Projective Geometry. Cambridge, U.K.: Oxford Univ. Press, 1952.

[10] F. Schwarzer, M. Saha, and J. Latombe, "Exact collision checking of robot paths," in Algorithmic Foundations of Robotics V. ser. Springer Tracts in Advanced Robotics, J. Boissonnat, J. Burdick, K. Goldberg, and S. Hutchinson, Eds. New York: Springer, 2004, pp. 25-41.

[11] E. Ferré and J.-P. Laumond, "An iterative diffusion algorithm for part disassembly," in Proc. IEEE Conf. Robot. Autom., New Orleans, LA, Apr. 2004, pp. 3149-3154.

[12] S. Cameron, "Collision detection by four-dimensional intersection testing," IEEE Trans. Robot. Automat., vol. 6, no. 3, pp. 291-302, Jun. 1990.
[13] S. Redon, A. Kheddar, and S. Coquillart, "Fast continuous collision detection between rigid bodies," Comput. Graph. Forum, vol. 21, no. 3, pp. 279-288, 2002.

[14] S. Redon, Y. J. Kim, M. C. Lin, D. Manocha, and J. Templeman, "Interactive and continuous collision detection for avatars in virtual environments," in Proc. IEEE Virtual Reality Conf., Chicago, IL, Mar. 2004, pp. $117-124$.

[15] S. Redon, M. C. Lin, D. Manocha, and Y. J. Kim, "Fast continuous collision detection for articulated models," ASME J. Comput. Inf. Sci. Eng., vol. 5, no. 2, pp. 126-137, 2005.

[16] W. Wang, J. Wang, and M.-S. Kim, "An algebraic condition for the separation of two ellipsoids," Comput. Aided Geom. Des., vol. 18, no. 6, pp. 531-539, 2001.

[17] Y.-K. Choi, W. Wang, and M.-S. Kim, "Exact collision detection of two moving ellipsoids under rational motions," in Proc. IEEE Conf. Robot. Autom., Taipei, Taiwan, R.O.C., Sep. 2003, pp. 349-354.

[18] H. Levy, Projective and Related Geometries. New York: Macmillan, 1964.

[19] L. E. Dickson, Elementary Theory of Equations. New York: Wiley, 1914.

[20] T. Horsch and B. Jüttler, "Cartesian spline interpolation for industrial robots," Comput. Aided Des., vol. 30, no. 3, pp. 217-224, 1998.

[21] B. Jüttler and M. G. Wagner, "Computer-aided design with spatial rational B-spline motions," ASME J. Mech. Des., vol. 118, no. 2, pp. 193-201, 1996.

[22] - "Kinematics and animation," in Handbook of Computer Aided Geometric Design, M. K. G. Farin and J. Hoschek, Eds. New York: Elsevier, 2002, pp. 723-748.

[23] O. Röschel, "Rational motion design-A survey," Comput. Aided Des., vol. 30, no. 3, pp. 169-178, 1998.

[24] M. G. Wagner, "Planar rational B-spline motions," Comput. Aided Des., vol. 27, no. 2, pp. 129-137, 1995.

[25] R. T. Farouki and V. T. Rajan, "On the numerical condition of polynomials in Bernstein form," Comput. Aided Geom. Des., vol. 4, no. 3, pp. 191-216, 1987.

[26] T. W. Sederberg, "Applications to computer aided geometric design," in Proc. AMS Symp. Appl. Math., vol. 53, D. Cox and B. Sturmfels, Eds., 1998, pp. 67-89.

[27] R. T. Farouki, "On the stability of transformations between power and Bernstein polynomial forms," Comput. Aided Geom. Des., vol. 8, no. 1, pp. 29-36, 1991

[28] H. M. Möller, "Counting zeros of polynomials by their Bézier ordinates," Univ. Dortmund, Dortmund, Germany, Ergebnisbericht Nr. 251, 2004.

[29] G. Alefeld and J. Herzberger, Introduction to Interval Computations. New York: Academic, 1983. translated by J. Rokne from orig. German "Einführung In Die Intervallrechnung".

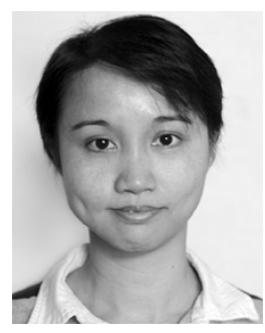

Yi-King Choi received the B.Sc. degree in 1996, and the M.Phil. degree in 2000, both in computer science, from the University of Hong Kong, Hong Kong, where she is currently working toward the Ph.D. degree.

Her research interests include computer graphics, geometric computing, and medical visualization.

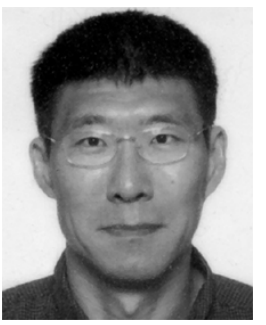

Wenping Wang received the B.Sc. and M.Eng. degrees in computer science from Shandong University, Shandong, China, in 1983 and 1986, respectively, and the Ph.D. degree in computer science from the University of Alberta, Edmonton, AB, Canada, in 1992.

$\mathrm{He}$ is currently an Associate Professor of Computer Science with the University of Hong Kong, Hong Kong. His research interests include computer graphics, geometric computing, and computational geometry. 


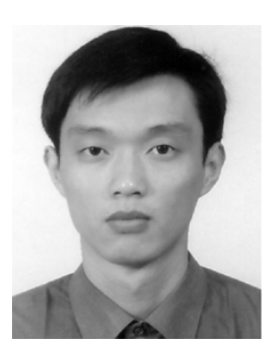

Yang Liu received the B.S. degree in 2000 , and the M.S. degree in 2003, both in mathematics, from the University of Science and Technology of China, Hefei, China. Currently, he is working toward the $\mathrm{Ph} . \mathrm{D}$. degree at the University of Hong Kong, Hong Kong.

His research interests include computer-aided design, computer graphics, and computational algebraic geometry.

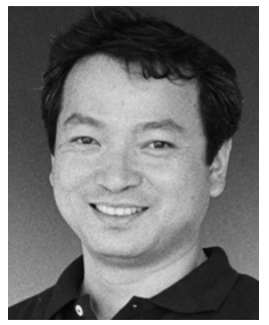

Myung-Soo Kim received the B.S. and M.S. degrees from Seoul National University, Seoul, Korea, in 1980 and 1982, respectively, and the M.S. degree in applied mathematics in 1985 and M.S. and Ph.D. degrees in computer science in 1987 and 1988, respectively, from Purdue University, West Lafayette, IN.

Currently, he is a Professor and the Head of the School of Computer Science and Engineering, Seoul National University, Seoul, Korea. His research interests are in computer graphics and geometric modeling. From 1988 to 1998, he was with the Department of Computer Science, Pohang University of Science and Technology (POSTECH), Pohang, Korea. He serves on the editorial boards of the journals Computer-Aided Design, Computer Aided Geometric Design, Computer Graphics Forum, and the International Journal of Shape Modeling. He also edited several special issues of journals including Computer-Aided Design, Graphical Models, the Journal of Visualization and Computer Animation, The Visual Computer, and the International Journal of Shape Modeling. Recently, together with G. Farin and J. Hoschek, he edited the Handbook of Computer Aided Geometric Design (Amsterdam, The Netherlands: North-Holland, 2002). 